OPEN ACCESS

Edited by:

Cheng Zhong,

Tianjin University, China

Reviewed by:

Bin Liu,

Tianjin University, China

Huanan Duan,

Shanghai Jiao Tong University, China

*Correspondence:

Awais Sattar Ghour

ghouriasg@gmail.com

Specialty section:

This article was submitted to

Energy Materials,

a section of the journal

Frontiers in Materials

Received: 28 December 2019

Accepted: 26 February 2020

Published: 26 March 2020

Citation:

Ghouri AS, Aslam R, Siddiqui MS and Sami SK (2020) Recent Progress

in Textile-Based Flexible

Supercapacitor. Front. Mater. 7:58.

doi: $10.3389 /$ fmats.2020.00058

\section{Recent Progress in Textile-Based Flexible Supercapacitor}

\author{
Awais Sattar Ghouri ${ }^{*}$, Rabya Aslam², Muhammad Saqib Siddiqui ${ }^{3}$ and \\ Syed Kamran Sami ${ }^{1}$
}

${ }^{1}$ Department of Chemical Engineering, Faculty of Engineering \& Architecture, Balochistan University of Information Technology, Engineering and Management Sciences, Quetta, Pakistan, ${ }^{2}$ Institute of Chemical Engineering \& Technology, University of the Punjab, Lahore, Pakistan, ${ }^{3}$ Department of Textile Engineering, Faculty of Engineering \& Architecture, Balochistan University of Information Technology, Engineering and Management Sciences, Quetta, Pakistan

In the backdrop of the growing requirement of flexible and wearable energy storage systems, textile-based supercapacitors having characteristic flexibility, superior charging-discharging rates, and low cost are ideal energy storage devices for wearable applications. Lightweight and flexible textile-based supercapacitors characterized by high conductivity, thermal, and environmental stability with negligible degradation under repeated use are required for multifunctional wearable electronics. Herein, supercapacitor based upon textile fabrics will be reviewed from the perspective of electrochemical, mechanical, and thermal properties without compromising flexibility, durability, and comfort of textile fabric.

Keywords: flexible supercapacitor, textile fabric, wearable applications, energy storage, conducting polymer

\section{INTRODUCTION}

Today, the world is facing overwhelming concerns pertaining to environmental pollution and energy shortage due to increased demand for energy consumption. The fulfillment of the energy needs by continuous reliance on conventional fossil fuels has reduced the energy sources significantly (González et al., 2016). These problems can be very well managed by proper utilization of clean and renewable energy sources like geothermal energy, wind energy, solar energy, and ocean energy but require feasible geographical distribution coupled with reliable, durable, efficient, and cost-effective energy storage technology (Xu et al., 2019).

In this regard, batteries are deemed as a promising alternative for power sources and energy storage systems. Although batteries have good energy capabilities but suffer from a series of drawbacks such as sluggish irreversible chemical reaction, low specific power, poor cycling performance, and inferior charge-discharge rate capability (González et al., 2016; Muzaffar et al., 2019; Yu and Feng, 2019). For flexible and wearable healthcare and portable electronic devices, supercapacitors have emerged as a superior alternative having enhanced energy storage capability ranging from a hundred to many thousands in the same volume as compared to batteries (Lee et al., 2013; González et al., 2016). Although the power output of the supercapacitor is comparatively low but possesses higher specific energy than a traditional electrolytic capacitor. A supercapacitor is bridging up the performances of both electrolytic capacitors and batteries. Supercapacitor offers long charge-discharge cycling stability and can withstand millions of cycles with sound coulombic efficiency without much decay in performances (González et al., 2016; Cheng et al., 2018; Muzaffar et al., 2019; Yu and Feng, 2019). 
Rapid development in modern digital communication, smart electronic devices for healthcare and wearable applications have augmented demand toward the formulation and design of improved and better flexible energy storage systems. To further enhance the characteristics of a supercapacitor, extensive efforts have been dedicated to the facile fabrication of supercapacitors with advanced materials (Lima et al., 2019).

Meanwhile, Fiber and fabric-based materials are being researched and employed as potential ingredients for supercapacitor applications. Textile-based flexible supercapacitors are lightweight, flexible, economical, and viable alternative to their conventional rigid and bulky counterparts. Due to having congenital flexibility and small volume, textilebased can be transformed into various shapes and structures, which in turns supports its integration with miniaturized futuristic wearable electronic devices and gadgets through well-established textile manufacturing technologies (Lee et al., 2013; Gulzar et al., 2016; Hong et al., 2019).

\section{TEXTILE-BASED FLEXIBLE SUPERCAPACITOR}

To cope up with the issues related to flexibility, wearability, and durability, research in respect of making the combination of supercapacitors with flexible textile fabric is bringing a revolution in our lives due to its lightweight, costeffectiveness, human-safe usage, flexibility, comfort, and good electrochemical performance.

Concomitantly, utilization of flexible substrate for supercapacitor fabrication with the capacity to accommodate stretching, deformation, and bending is springing up with a rapid pace and potential utilization of new and advanced materials with diverse properties is being explored and harnessed.

In consequence, the production of more efficient textilebased supercapacitors with a number of novel features and functions must be focused for next-generation flexible electronics (Liu et al., 2015).

A new asymmetric supercapacitor prepared from a composite of horizontally aligned carbon nanotube array (HACNTs) and poly (3-methylthiophene) (P3MT) depicted high areal capacitance of $3.1 \mathrm{~F} \mathrm{~cm}^{-2}$ at $5 \mathrm{~mA} \mathrm{~cm}^{-2}$ and sustained the areal capacitance of $1.8 \mathrm{~F} \mathrm{~cm}^{-2}$ even at the current density of $200 \mathrm{~mA} \mathrm{~cm}^{-2}$. Although little structural change observed during the charge/discharge process due to change in volume of conducting polymer under high strain in as-prepared supercapacitor performance but shows good electrochemical and mechanical stability of electrode material (Zhou et al., 2019). Cheng et al. prepared high-performance flexible supercapacitor consisting of PANI/SWCNT/cloth composite electrode, which delineated specific capacitance of $410 \mathrm{Fg}^{-1}$ and good cycle life and stability similar to other electrodes (Wang et al., 2011). The energy density of $6.6 \mathrm{~W} \mathrm{~h} \mathrm{~kg}^{-1}$ is being observed at power density of $7,000 \mathrm{~W} \mathrm{~kg}$. It is important to note that, at the same power density PANI/SWCNT/cloth depicts high energy density, rate capability, stability, and capacitance than supercapacitor made from PANI/cloth and SWCNT/cloth-based electrode. The obtained results enlighten the potential utilization of PANI/SWCNT/clothbased supercapacitor for flexible and wearable applications (Cheng et al., 2018).

Cotton cloth is known for its intrinsic porosity, hydrophilicity, and flexibility. A low-cost all-solid-state and flexible supercapacitor using cotton cloth has been developed by Wang et al. via the facile electroplating method. In their work, cotton cloth is initially made conductive by sputtering 99.99\% pure Copper $(\mathrm{Cu})$ and subsequent electrodeposition of tremella-like nickel-cobalt-sulfide (Ni-Co-S) nanoflakes on it. An asymmetric supercapacitor containing Ni-Co-S as the cathode, activated carbon as the anode, and PVA/KOH as the solid-state electrolyte has been fabricated. The as-fabricated asymmetric flexible supercapacitor displayed the energy density of $48.9 \mathrm{~W} \mathrm{~h} \mathrm{~kg}^{-1}$ at $390 \mathrm{~W} \mathrm{~kg}-1$ with capacitance retention of $70 \%$ after 5,000 cycles. The developed textile-based asymmetric flexible supercapacitor with good rate capability, bendability, and flexibility possesses practical applications in wearable energy storage devices (Wang et al., 2020).

\section{WEARABLE APPLICATIONS OF TEXTILE-BASED FLEXIBLE SUPERCAPACITOR}

Applicability of supercapacitor in the field of wearable electronics with a diverse application is being focused on research works with an aim to explore its energy storage performance. Most recently, high-performance textile electrodes by applying a coating of polypyrrole (PPy) using improved in situ polymerization technique have been developed with a resistance less than $10 \Omega \mathrm{cm}^{-2}$ and the highest capacitance of $4848 \mathrm{mF} \mathrm{cm} \mathrm{cm}^{-2}$ at $1 \mathrm{~mA} \mathrm{~cm}^{-2}$. Increased resistance of fabric was observed using water washing but depicted unchanged conductivities using dichloromethane as a washing agent even after 20 laundering cycles. Further, no alteration in conductivity occurred during mechanical deformation, sewing, and cutting process (Lv et al., 2019b).

Similarly, in their subsequent work, polypyrrole (PPy) was coated on knitted cotton fabric using improved in situ chemical polymerization technique. Three samples were prepared using a molar ratio of pyrrole monomer to ferric trichloride hexahydrate $\left(\mathrm{FeCl}_{3} .6 \mathrm{H}_{2} \mathrm{O}\right)$ of $0.5,1$, and 2 . The prepared textile electrode samples were named as $\mathrm{PPy}-0.5, \mathrm{PPy}-1$, and $\mathrm{PPy}-2$, respectively. As compared to PPy-0.5 and PPy-1, PPy-2 textile electrode exhibited superior conductivity and high mass loading of active material. PPy-2 fabric electrode showed specific areal capacitance of $5073 \mathrm{mF} \mathrm{cm}^{-2}$ at $1 \mathrm{~mA} \mathrm{~cm}^{-2}$ with excellent flexibility and cycle stability. In addition, an all-solid-state symmetric supercapacitor was fabricated using two PPy-2 fabric electrodes, LiCl/PVA gel electrolyte, a cellulosic separator (TF4030, NKK), and two silver foils. The fabricated symmetric all-solid-state supercapacitor demonstrated high specific areal capacitance of $1167.9 \mathrm{mF} \mathrm{cm}^{-2}$ and $904.2 \mathrm{mF} \mathrm{cm}^{-2}$ at $1 \mathrm{~mA} \mathrm{~cm}^{-2}$ and $20 \mathrm{~mA} \mathrm{~cm}^{-2}$, respectively. The supercapacitor has displayed energy density and power density of $102.4 \mu \mathrm{W} \mathrm{cm}^{-2}$ and 
$0.39 \mathrm{mWcm}^{-2}$, respectively. The resultant electrochemical performance enables the transformation of textile-based supercapacitors into wearable energy storage devices (Lv et al., 2019a).

All-solid-state flexible supercapacitor based upon carbon woven fabric/PANI/graphene composite having appealing potential for wearable applications has been fabricated using in situ growth and wrapping method, displaying a high areal capacitance $\left(790 \mathrm{~F} \mathrm{~cm}^{-2}\right)$ at a current density of $1 \mathrm{~A} \mathrm{~cm}^{-2}$ and

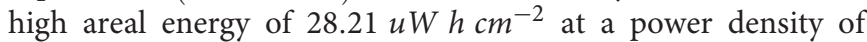
$0.12 \mathrm{~mW} \mathrm{~cm}^{-2}$ under bending conditions. In the as-assembled flexible supercapacitor, graphene improved the flexibility, performance, and conductivity of the electrode. In addition, rGO nanosheets combined well with PANI particle limiting volume change during charge/discharge cycles by exhibiting enhanced stability with $88.9 \%$ capacitance retention (Lin et al., 2018). In the work of Ismail and coworkers, a flexible electrode for supercapacitor having cycling stability up to 35,000 cycles have been synthesized by dipping and drying method using carbon nanotubes cotton super-fiber. The CNT cotton super-fiber can be twisted and woven into fiber and fabric, respectively. The prepared CNT cotton super-fiber based supercapacitor has superior flexibility, strength, electric, and thermal conductivity (Ismail et al., 2019).

All-solid-state supercapacitor (ASSSC) having great potential for wearable energy storage devices has been developed by Wang and coworkers by facile in situ chemical polymerization method using polypyrrole and knitted cotton fabric as substrate. ASSSC exhibited good stretching stability and hydrophobicity with a water-contact-angle above $133^{\circ}$. The ASSSC displayed areal capacitance of $450 \mathrm{mF} \mathrm{cm} \mathrm{cm}^{-2} @ 5 \mathrm{mV} \mathrm{s}^{-1}$ and $1 \mathrm{~mA} \mathrm{~cm}^{-2}$, energy density and power density of $0.4 \mathrm{Wh} \mathrm{cm}^{-2}$ and $10 \mathrm{~W} \mathrm{~m}^{-2}$, respectively. Even under $40 \%$ strain, stretching of 1,000 times, ASSSC maintained initial capacitance about 78-91\% at different current densities and depicted an increase in areal capacitance of 160\%@5 $\mathrm{mA} \mathrm{cm}^{-2}$. Apart from above, ASSC set backed in cycling stability using gel electrolyte primarily due to expulsion of moisture from gel electrolyte, resulting in the retention of $30 \%$ of initial capacitance after 500 cycles. On the other hand, retention of $53 \%$ of initial capacitance at 5,000th cycles was observed, when aqueous electrolyte was used (Wang et al., 2019a). Textile-based flexible supercapacitor for wearable application has been developed by Lima and coworkers using graphene nanoplatelets/CNT/polypyrrole composite on cotton electrode exhibiting good cyclability, high specific capacitance of $45.5 \mathrm{~F} \mathrm{~g}^{-1}$, and capacitance retention of $70 \%$ after 2,000 cycle of use. The electrochemical performance of the prepared flexible supercapacitor is better than $\mathrm{CNT} /$ polypyrrole composite-based flexible supercapacitor. Further, varying relative proportions of GNP/CNT ratio also improved the electrochemical performance of as-prepared flexible supercapacitor (Lima et al., 2019).

A hierarchical wearable solid-state supercapacitor yarn for electrode preparation with excellent volumetric energy density and power density has been synthesized using graphenemetallic textile composite. The prepared $\mathrm{rGo} / \mathrm{Ni}$ cotton yarn presented enhanced combined capacitance, high energy and power density, exceptional mechanical durability, and superior flexibility having huge potential for scalability because of ease of material synthesis and device fabrication. Using embroidery and weaving techniques, $\mathrm{rGo} / \mathrm{Ni}$ cotton yarn can be integrated into the wearable application. The $\mathrm{rGo} / \mathrm{Ni}$ cotton yarn supersedes the electrochemical performance of other available yarn-based supercapacitor due to having a large surface area of active material and providing enough excess to the electrolyte to form an electric double layer. In principle, the fabricated $\mathrm{rGo} / \mathrm{Ni}$ cotton yarn-based supercapacitor can be well amalgamated with other textile scaffolds and active materials and can be used as effective and efficient energy-storage devices for next-generation flexible, portable, and wearable electronics (Liu et al., 2015).

Sami et al. fabricated lightweight and flexible all-solid-state 1D-1D hybrid supercapacitor composed of zinc oxide nanorods @ PEDOT:PSS coated electrospun P(VDF-TrFE) nanofibers exhibiting gravimetric specific capacitance of $59.55 \mathrm{Fg}^{-1}$ and $56.99 \mathrm{~F} \mathrm{~g}^{-1}$ determined from cyclic voltammogram and galvanostatic charge-discharge curve. The high areal capacitance of $1.22 \mathrm{mF} \mathrm{cm}^{-2}$ at the current density of $0.1 \mathrm{~mA} \mathrm{~cm}^{-2}$ indicates the possibility of developing a next-generation integrated flexible power source for wearable applications. Moreover, excellent rate capability and mechanical robustness ascertain its usability for high performance flexible energy storage devices (Sami et al., 2017). A flexible textile-based supercapacitor with good flexibility and specific capacitance of $578 \mathrm{~F} \mathrm{~g}^{-1}$ at scan rate of $5 \mathrm{mV} \mathrm{s}^{-1}$ has been developed by incorporating vertical array of Co-Ni layered double hydroxide $(\mathrm{Co}-\mathrm{Ni} \mathrm{LDH})$ on conductive graphene/cotton fabric (G/CF) by a chemical deposition method. The prepared Co-Ni LDH/G/CF has a significant impact on developing flexible energy storage devices for wearable applications (Wang et al., 2019b).

\section{DESIGN STRATEGIES FOR TEXTILE-BASED FLEXIBLE SUPERCAPACITOR}

Textile fabric can be made conductive by applying a suitable coating of conducting polymers on them. Conductive fabric offers good electrochemical properties and is therefore well suited for diverse applications ranging from smart textiles to energy storage purposes. This is because conventional textiles have their own innate breathability, pliability, conformability, and wearability. Recently, a simple and scalable interface engineering processing has been developed for exfoliating graphene nanoplatelets (GNP) on polyamide fabric using a low boiling solvent. After $4 \mathrm{~h}$ of coating, the resultant GNP/polyamide fabric displayed a decrease in surface resistivity from infinity $(\infty)$ to $40 k \Omega$. Further, the minimal effect on fabric crystallinity and uniform coating of GNP nanoplatelets on polyamide fabric has been confirmed by XRD and SEM results, respectively. This interface engineering approach is used for coating any polymeric fabric to produce E-textile related material (Barjasteh et al., 2019).

Several flexible heating devices based upon polypyrrole/cotton fabric are being prepared by variable pyrrole/ $\mathrm{FeCl}_{3}$ molar concentrations. It has been noted that flexible heating 


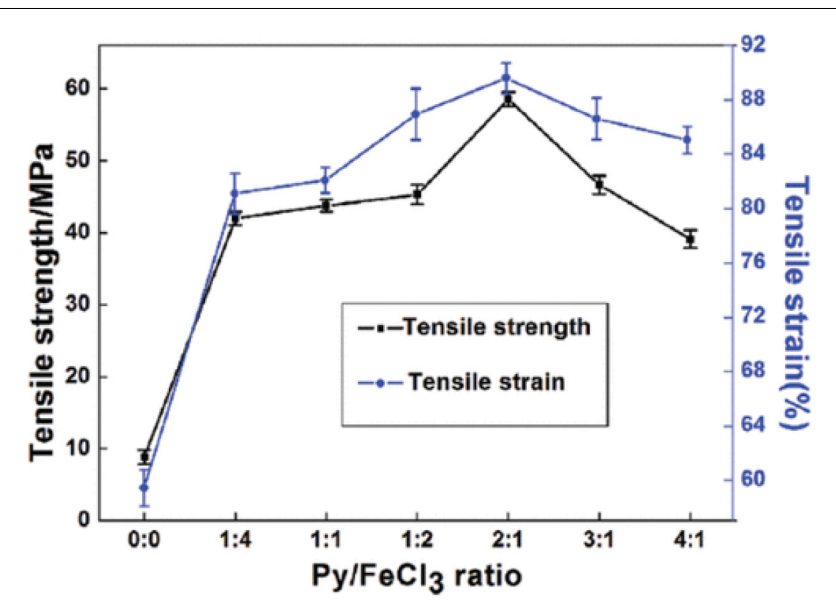

FIGURE 1 | Tensile properties of raw cotton (warp) and PPy/cotton composites with different Py/FeCl3 ratios. Reproduced from Xie et al. (2019) under the Creative Commons CC-BY license.

devices displayed low resistivity, i.e., $0.37 \Omega \mathrm{cm}$, better tensile strength than raw cotton fabric, i.e., $58 \mathrm{MPa}$ (as shown in Figure 1). In contrast to the tensile strength of the raw cotton fabric, i.e., 8.9 $\mathrm{MPa}$, the improvement in tensile strength of PPy/composites, i.e., $58 \mathrm{MPa}$ is not significantly affected by $\mathrm{Py} / \mathrm{FeCl}_{3}$ concentration ratios but is attributed to the attachment of PPy on the cotton fabric. Further, the electrothermal performance of prepared PPy/cotton composites was presented in the temperature-time curve of Figure $\mathbf{2}$ under different $\mathrm{Py} / \mathrm{FeCl}_{3}$ concentration ratios and voltage inputs $(1-16 \mathrm{~V})$. It is depicted from the temperature-time curve in Figure 2 that the temperature of $\mathrm{PPy} /$ cotton composites rises sharply within few seconds under certain voltage input, and after attaining peak value, the temperature remains almost constant throughout the heating period. The higher the temperature rise, the slower it drops to room temperature. It is noted that $\mathrm{PPy} /$ cotton composites quickly reach the temperature value of $168.3^{\circ} \mathrm{C}$ after $3 \mathrm{~min}$ at the applied voltage of $5 \mathrm{~V}$, whereas, the capability of $\mathrm{PPy} /$ cotton composites strongly depends upon high conductivity under certain input voltage. In the context of the above results, the flexible heating devices exhibit good electric, mechanical, and thermal properties. It is also expected that an as-prepared flexible heating device can be well integrated with the fabric-based supercapacitor (Xie et al., 2019).

In addition, the utilization of flexible and wearable supercapacitor in electronic textile and energy storage system is on the upswing. In contrast to conductive fabric, fibers, threads, and yarns are also being made conductive by means of applying the coating of conducting polymers using numerously available and well-established coating techniques. As consistent with above, Lee et al. have prepared electrode material having high energy density, power density, and cycle life for redox supercapacitor using a novel gradient biscrolling process. In this technique, hundreds of carbon nanotubes infiltrated by conducting polymers were scrolled into $\sim 20 \mu \mathrm{m}$ diameter yarn. Subsequently, scrolled yarn was plied with metal wires to enhance its power generation capability. The resultant electrode material for supercapacitor exhibited volumetric capacitance of $\sim 179 \mathrm{~F} \mathrm{~cm}^{-3}$ and discharge current for the plied yarn displayed a linear rise with a voltage scan rate of $\sim 20 \mathrm{Vs}^{-1}$ and $\sim 80 \mathrm{Vs}^{-1}$ for solid and liquid electrolytes, respectively (Lee et al., 2013).

Successful commercialization of smart electronic textile and textile-based supercapacitor relies upon flexible and wearable substrates, whereas, traditional electrochemical energy storage devices are too bulky and rigid to be used for the said purpose. Much of the practical efforts have been dedicated to developing fiber/fabric-based supercapacitor but, still needs focused work in respect of supercapacitor stability, scalability, wearability, and fabrication efficiency. From this perspective, the onestep wet spinning method with a high degree of production rate, i.e., $118 \mathrm{~m} / \mathrm{h}$ for developing supercapacitor fiber having sound electrochemical properties has been proposed by Hong et al. The unique two-circle-in-one-circle structural design of supercapacitor fiber displays good electrochemical stability on bending for $10^{5}$ cycles and can be woven into a flexible and wearable power scarf and fabric. In general, this facile and scalable synthesis approach can pave the way for designing fiber-shaped healthcare devices, solar cells, batteries, sensors, etc (Hong et al., 2019).

For the first time, Cheng et al. have reported the fabrication of novel textile-based flexible supercapacitor using a conventional dyeing process following the coating of PEDOT:PSS on PET fabric. In this study, organic dyes, i.e., C.I. Disperse Blue 79 (DB 79) and C.I. Disperse Red 60 (DR 60) was absorbed on the PET fabric using a well-known textile dyeing process to improve capacitance performance of the fabric, whereas, the poly (3,4-ethylenedioxythiophene):poly(styrenesulfonate) (PEDOT:PSS) solution was coated on dyed PET fabric by the spray-coating method to enhance the conductivity of the fabric. It was noted that the introduction of organic dyes on the PET fabric can ameliorate the electrochemical performance of PEDOT:PSS-coated PET Fabric. It is worth noting that DB 79 improved the areal capacitance of fabric from $50.92 \mathrm{mF} \mathrm{cm} \mathrm{cm}^{-2}$ to $74.99 \mathrm{mF} \mathrm{cm}^{-2}$ at the current density of $0.5 \mathrm{~mA} \mathrm{~cm}$. Meanwhile, the assembled symmetric supercapacitor based upon PEDOT:PSS@DR 60@PET fabric electrode displayed the energy density of $4.10 \mathrm{~mW} \mathrm{hm}^{-2}$ at $400.03 \mathrm{~mW} \mathrm{~cm}^{-2}$ with capacitance retention of $87 \%$ after 200 cycles. The assembled all-organic supercapacitor can power an LED, demonstrating its potential application in wearable electronics (Cheng et al., 2020).

Commercial polyester conductive fabric coated with nickel and copper (PCF) is used as a composite electrode for flexible supercapacitor by Qi and coworkers. In this work, Qi et al. firstly prepared composite fabric electrode by physically depositing graphene oxide (GO) followed by thermal and ascorbic acidassisted reduction of graphene oxide. Subsequently, a uniform layer of nickel-cobalt layered double hydroxide (Ni-Co LDH) having $1.4 \mu \mathrm{m}$ thickness is electrodeposited on reduced graphene oxide (rGo) surface. The NiCo-LDH@rGo PCF composite electrode exhibits specific capacitance of $1220.5 \mathrm{Fg}^{-1}$ at the current density of $1 \mathrm{Ag}^{-1}$ with capacitance retention of $84.1 \%$ 

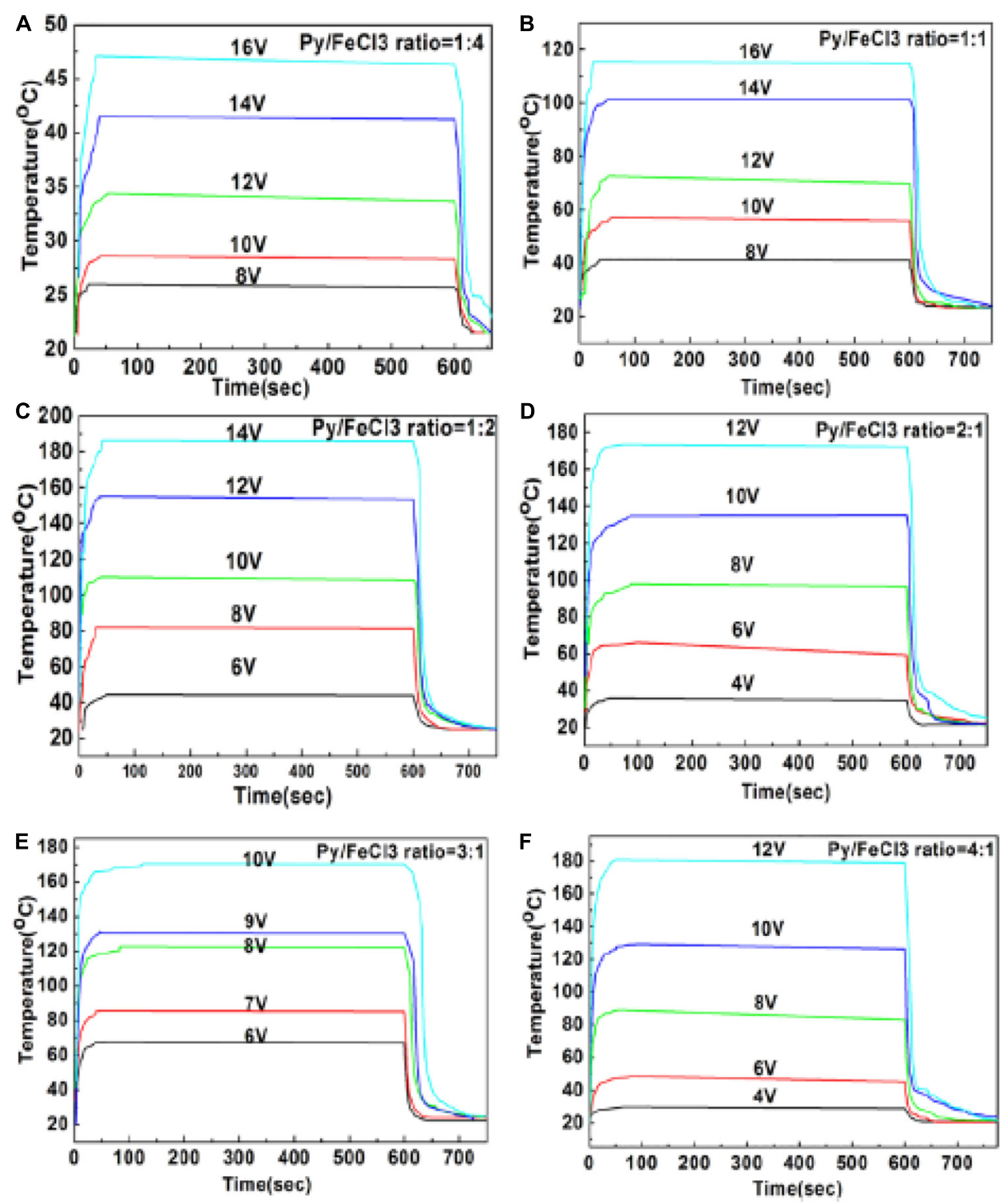

FIGURE 2 | Temperature-time curve changes of PPy/cotton composites with different Py/FeCl3 ratios (A) 1:4, (B) 1:1, (C) 1:2, (D) 2:1, (E) 3:1, and (F) 4:1 at different applied voltages of 1-16 V. Reproduced from Xie et al. (2019) under the Creative Commons CC-BY license.

after 5,000 cycles at the current density of $8 \mathrm{Ag}^{-1}$. An asymmetric flexible supercapacitor containing $\mathrm{Fe}_{2} \mathrm{O}_{3} / \mathrm{N}-\mathrm{rGo}$ as the cathode, NiCo-LDH@rGo PCF as the anode, and PVA-KOH as the solid-state electrolyte have been fabricated. The as-fabricated asymmetric flexible supercapacitor displayed the energy density of $61.1 \mathrm{Wh} \mathrm{kg}^{-1}$ at $856.1 \mathrm{Wh} \mathrm{kg}^{-1}$ with remarkable cycling stability of $90.6 \%$ capacitance retention after 5,000 cycles. The prepared asymmetric flexible supercapacitor has a significant impact on developing flexible energy storage devices for wearable applications (Qi et al., 2020). To this end, commendable efforts have been dedicated in the fabrication of flexible supercapacitor electrodes based upon waste cotton fabric, carbon woven fabric, carbonized cotton fabric, activated carbon fabric by many researchers for high energy storage applications
(González et al., 2016; Kim et al., 2018; Ippili et al., 2019; Jin et al., 2019; Lin et al., 2019; Reece et al., 2019; Wang et al., 2019c; Xu et al., 2019).

\section{CONCLUSION AND FUTURE PERSPECTIVES}

Keeping in view widespread applications of flexible supercapacitor in wearable electronics, modification methodologies in respect of fabrication, electrochemical performance, choosing reliable and smart advanced materials, electrolyte selection, etc. are being discovered to bring forward lightweight and flexible supercapacitor owing high energy 
and power density, safe and easy operation in conjunction with good interface with human posture (Lin et al., 2018). Therefore, the need of the time is to develop a cost-effective and efficient energy storage system by keeping in view the latest available technological aspects, which can deliver high power and high energy rates. In view of the above, still, the textile-based fabric has the capability of being further explored and tested as a good ingredient for supercapacitors. Previously, textilebased supercapacitors having both synergetic effects of electric double-layer capacitance combined with pseudocapacitance of conducting polymers have not been reported yet. Although, work has been done regarding incorporation of conducting polymer on polyimide (PI) matrix, which gave better results related to thermal, mechanical, environmental stability along with excellent electroactivity (Arbizzani et al., 1996; González et al., 2016), but the same composite comprised of conductive polymers has not been tested upon any textile fabric for supercapacitor applications. In addition, the incorporation of stretchable electrodes having improved electrochemical performance of power/energy density must be focused for tangible outcomes. Low energy density observed in wearable textile-based flexible supercapacitors can be well addressed

\section{REFERENCES}

Arbizzani, C., Mastragostino, M., and Meneghello, L. (1996). Polymer-based redox supercapacitors: a comparative study. Electrochim. Acta 41, 21-26. doi: 10.1016/ 0013-4686(95)00289-Q

Barjasteh, E., Sutanto, C., and Nepal, D. (2019). Conductive polyamide-graphene composite fabric via interface engineering. Langmuir 35, 2261-2269. doi: 10 . 1021/acs.langmuir.8b03543

Cheng, M., Meng, Y.-N., and Wei, Z.-X. (2018). Conducting polymer nanostructures and their derivatives for flexible supercapacitors. Israel J. Chem. 58, 1299-1314. doi: 10.1002/ijch.201800077

Cheng, Q., Meng, C., Qian, Y., He, J., and Dong, X. (2020). Energy capacity enhancement of all-organic fabric supercapacitors by organic dyes: old method for new application. Prog. Org. Coat. 138:105439. doi: 10.1016/j.porgcoat.2019. 105439

González, A., Goikolea, E., Barrena, J. A., and Mysyk, R. (2016). Review on supercapacitors: technologies and materials. Renew. Sustain. Energy Rev. 58, 1189-1206. doi: 10.1016/j.rser.2015.12.249

Gulzar, U., Goriparti, S., Miele, E., Li, T., Maidecchi, G., Toma, A., et al. (2016). Next-generation textiles: from embedded supercapacitors to lithium ion batteries. J. Mater. Chem. 4, 16771-16800. doi: 10.1039/c6ta0 $6437 \mathrm{j}$

Hong, Y., Cheng, X.-L., Liu, G.-J., Hong, D.-S., He, S.-S., Wang, B.-J., et al. (2019). One-step production of continuous supercapacitor fibers for a flexible power textile. Chin. J. Polym. Sci. 37, 737-743. doi: 10.1007/s10118-019-2301-5

Ippili, S., Jella, V., Eom, J.-H., Kim, J., Hong, S., Choi, J.-S., et al. (2019). An eco-friendly flexible piezoelectric energy harvester that delivers high output performance is based on lead-free MASnI3 films and MASnI3PVDF composite films. Nano Energy 57, 911-923. doi: 10.1016/j.nanoen.2019. 01.005

Ismail, I., Md Yusof, J., Mat Nong, M. A., and Adnan, N. L. (2019). "Synthesis of carbon nanotube-cotton superfiber materials," in Synthesis, Technology and Applications of Carbon Nanomaterials, eds S. A. Rashid, R. N. I. R. Othman, and M. Z. Hussein (Amsterdam: Elsevier), 61-76. doi: 10.1016/B978-0-12-8157572.00003-6

Jin, K., Zhou, M., Zhao, H., Zhai, S., Ge, F., Zhao, Y., et al. (2019). Electrodeposited $\mathrm{CuS}$ nanosheets on carbonized cotton fabric as flexible supercapacitor electrode for high energy storage. Electrochim. Acta 295, 668-676. doi: 10.1016/j.electacta. 2018.10.182 by extending the working potential window, which can be done by exploring new and improved electrolytes, whereas, appropriate chemical modification of electrode can enhance specific capacitance of textile-based flexible supercapacitor (Lima et al., 2019). Preparation of ameliorated conducting polymer composite having superior and tunable conductivities and flexibility is a feasible key factor in bringing about desired and improved electrochemical characteristics in textile-based flexible supercapacitor (Le et al., 2017).

\section{AUTHOR CONTRIBUTIONS}

AG, RA, MS, and SS contributed equally in mini review article write-up.

\section{ACKNOWLEDGMENTS}

I feel extremely grateful to my colleagues who supported me extensively throughout the work and provided their valuable and expert opinion on each topic.

Kim, J. H., Choi, C., Lee, J. M., de Andrade, M. J., Baughman, R. H., and Kim, S. J. (2018). Ag/MnO2 composite sheath-core structured yarn supercapacitors. Sci. Rep. 8:13309. doi: 10.1038/s41598-018-31611-2

Le, T. H., Kim, Y., and Yoon, H. (2017). Electrical and electrochemical properties of conducting polymers. Polymers 9:150. doi: 10.3390/polym9040150

Lee, J. A., Shin, M. K., Kim, S. H., Cho, H. U., Spinks, G. M., Wallace, G. G., et al. (2013). Ultrafast charge and discharge biscrolled yarn supercapacitors for textiles and microdevices. Nat. Commun. 4:1970. doi: 10.1038/ncomms2970

Lima, R. M. A. P., de Oliveira, M. C. A., and de Oliveira, H. P. (2019). Wearable supercapacitors based on graphene nanoplatelets/carbon nanotubes/polypyrrole composites on cotton yarns electrodes. SN Appl. Sci. 1:325. doi: $10.1007 / \mathrm{s} 42452-019-0343-5$

Lin, X., Wu, M., Zhang, L., and Wang, D. (2019). Superior stretchable conductors by electroless plating of copper on knitted fabrics. ACS Appl. Electron. Mater. 1, 397-406. doi: 10.1021/acsaelm.8b00115

Lin, Y., Zhang, H., Deng, W., Zhang, D., Li, N., Wu, Q., et al. (2018). In-situ growth of high-performance all-solid-state electrode for flexible supercapacitors based on carbon woven fabric/ polyaniline/ graphene composite. J. Power Sources 384, 278-286. doi: 10.1016/j.jpowsour.2018.03.003

Liu, L., Yu, Y., Yan, C., Li, K., and Zheng, Z. (2015). Wearable energy-dense and power-dense supercapacitor yarns enabled by scalable graphene-metallic textile composite electrodes. Nat. Commun. 6:7260. doi: 10.1038/ncomms8260

Lv, J., Zhang, L., Zhong, Y., Sui, X., Wang, B., Chen, Z., et al. (2019a). Highperformance polypyrrole coated knitted cotton fabric electrodes for wearable energy storage. Org. Electron. 74, 59-68. doi: 10.1016/j.orgel.2019.06.027

Lv, J., Zhou, P., Zhang, L., Zhong, Y., Sui, X., Wang, B., et al. (2019b). High-performance textile electrodes for wearable electronics obtained by an improved in situ polymerization method. Chem. Eng. J. 361, 897-907. doi: 10.1016/j.cej.2018.12.083

Muzaffar, A., Ahamed, M. B., Deshmukh, K., and Thirumalai, J. (2019). A review on recent advances in hybrid supercapacitors: design, fabrication and applications. Renew. Sustain. Energy Rev. 101, 123-145. doi: 10.1016/j.rser.2018.10.026

Qi, J., Chen, Y., Li, Q., Sui, Y., He, Y., Meng, Q., et al. (2020). Hierarchical NiCo layered double hydroxide on reduced graphene oxide-coated commercial conductive textile for flexible high-performance asymmetric supercapacitors. J. Power Sources 445:227342. doi: 10.1016/j.jpowsour.2019.227342

Reece, R., Lekakou, C., and Smith, P. A. (2019). A structural supercapacitor based on activated carbon fabric and a solid electrolyte. Mater. Sci. Technol. 35, 368-375. doi: 10.1080/02670836.2018.1560536 
Sami, S. K., Siddiqui, S., Shrivastava, S., Lee, N.-E., and Chung, C.-H. (2017). The pine-needle-inspired structure of zinc oxide nanorods grown on electrospun nanofibers for high-performance flexible supercapacitors. Small 13:1702142. doi: 10.1002/smll.201702142

Wang, B., Song, W., Gu, P., Fan, L., Yin, Y., and Wang, C. (2019a). A stretchable and hydrophobic polypyrrole/knitted cotton fabric electrode for all-solid-state supercapacitor with excellent strain capacitance. Electrochim. Acta 297, 794804. doi: 10.1016/j.electacta.2018.12.042

Wang, K., Zhao, P., Zhou, X., Wu, H., and Wei, Z. (2011). Flexible supercapacitors based on cloth-supported electrodes of conducting polymer nanowire array/SWCNT composites. J. Mater. Chem. 21:16373. doi: 10.1039/c1jm13722k

Wang, Y., Ding, Y., Guo, X., and Yu, G. (2019b). Conductive polymers for stretchable supercapacitors. Nano Res. 12, 1978-1987. doi: 10.1007/s12274-0192296-9

Wang, Z., Wang, H., Ji, S., Wang, H., Brett, D. J. L., and Wang, R. (2020). Design and synthesis of tremella-like $\mathrm{Ni}$-Co-S flakes on co-coated cotton textile as high-performance electrode for flexible supercapacitor. J. Alloys Compd. 814:151789. doi: 10.1016/j.jallcom.2019.151789

Wang, Y.-F., Yi, S., and Bian, S.-W. (2019c). Flexible textile-based electrode materials for supercapacitors. Mater. Today Proc. 16, 1448-1455. doi: 10.1016/ j.matpr.2019.05.322

Xie, J., Pan, W., Guo, Z., Jiao, S. S., and Ping Yang, L. (2019). In situ polymerization of polypyrrole on cotton fabrics as flexible electrothermal materials. J. Eng. Fiber. Fabr. 14:155892501982744. doi: 10.1177/155892501982 7447

Xu, J., Li, X., Li, X., Li, S., Zhao, L., Wang, D., et al. (2019). Freestanding cotton-derived carbon microfiber@nickel-aluminum layered double hydroxides composite and its excellent capacitive performance. J. Alloys Compd. 787, 27-35. doi: 10.1016/j.jallcom.2019.01.270

Yu, M., and Feng, X. (2019). Thin-film electrode-based supercapacitors. Joule 3, 338-360. doi: 10.1016/j.joule.2018.12.012

Zhou, Y., Wang, X., Acauan, L., Kalfon-Cohen, E., Ni, X., Stein, Y., et al. (2019). Ultrahigh-areal-capacitance flexible supercapacitor electrodes enabled by conformal P3MT on horizontally aligned carbon-nanotube arrays. Adv. Mater. 31:1901916. doi: 10.1002/adma.201901916

Conflict of Interest: The authors declare that the research was conducted in the absence of any commercial or financial relationships that could be construed as a potential conflict of interest.

Copyright (C) 2020 Ghouri, Aslam, Siddiqui and Sami. This is an open-access article distributed under the terms of the Creative Commons Attribution License (CC BY). The use, distribution or reproduction in other forums is permitted, provided the original author(s) and the copyright owner(s) are credited and that the original publication in this journal is cited, in accordance with accepted academic practice. No use, distribution or reproduction is permitted which does not comply with these terms. 